\title{
EFFECTS OF ELECTRONS ON THE ELECTROMAGNETIC ION CYCLOTRON INSTABILITY: SOLAR WIND IMPLICATIONS
}

\author{
S. M. ShaAban ${ }^{1,2}$, M. Lazar ${ }^{1,3}$, S. Poedts $^{1}$, and A. Elhanbaly ${ }^{2}$ \\ ${ }^{1}$ Centre for Mathematical Plasma Astrophysics, Celestijnenlaan 200B, B-3001 Leuven, Belgium; shaaban.mohammed@ student.kuleuven.be \\ ${ }^{2}$ Theoretical Physics Research Group, Physics Department, Faculty of Science, Mansoura University, 35516, Egypt \\ ${ }^{3}$ Belgian Institute for Space Aeronomy 3-Avenue Circulaire, B-1180 Brussels, Belgium \\ Received 2015 July 1; accepted 2015 October 12; published 2015 November 13
}

\begin{abstract}
In diffuse plasmas in space, particle-particle collisions are rare and inefficient, such that a plausible mechanism for constraining the temperature anisotropy of plasma particles may be provided by the resulting instabilities. The implication of the electromagnetic ion-cyclotron (EMIC) instability in the solar wind is still unclear because this instability is fast enough to relax the proton temperature anisotropy, but the $1 \mathrm{AU}$ measurements do not conform to the instability thresholds predicted by the existing theories, which ignore the kinetic effects of electrons, assuming them to be isotropic. This paper presents a refined analysis of the EMIC instability in the presence of a temperature (T) anisotropy of electron (subscript "e") population, i.e., $A_{\mathrm{e}}=T_{\mathrm{e}, \perp} / T_{\mathrm{e}, \|} \neq 1$, enabling the identification of two distinct regimes of this instability that correspond to an excess of perpendicular temperature $\left(A_{\mathrm{e}}>1\right)$ or an excess of parallel temperature $\left(A_{\mathrm{e}}<1\right)$. The growth rates, real frequencies, and threshold conditions are found to be highly sensitive to the electron temperature anisotropy, and electrons with $A_{\mathrm{e}}>1$ inhibit the instability, while for $A_{\mathrm{e}}<1$ the instability growth rates increase with the electron anisotropy. Moreover, the electron-proton temperature ratio $\theta_{T}=T_{\mathrm{e}, \|} / T_{\mathrm{p}, \|}$ becomes an important factor that stimulates the effect of the anisotropic electrons. The potential relevance of the new results in the solar wind is analyzed by contrasting the instability thresholds with the observed limits of the proton temperature anisotropy.
\end{abstract}

Key words: instabilities - plasmas - solar wind

\section{INTRODUCTION}

Because space plasmas are hot and dilute Coulomb collisions are rare and inefficient for limiting the departures of charged particles from thermodynamic equilibrium. However, in the solar wind there are other important mechanisms, like adiabatic cooling, i.e., the CGL (according to Chew et al. 1956) or double adiabatic expansion (Matteini et al. 2007), anisotropic heating (by cyclotron-resonant dissipation of kinetic Alfvén waves transported by the solar wind) (Matteini et al. 2012), and kinetic instabilities (Gary 1993), which are expected to regulate the increase of free energy and explain the in situ observations (Hellinger et al. 2006; Stverák et al. 2008; Bale et al. 2009; Matteini et al. 2012). In a recent review, Matteini et al. (2012) provide a comprehensive description of these mechanisms, presenting evidence that the kinetic anisotropy of protons and heavier ions in the fast winds is governed by the adiabatic expansion (large-scale physics), while kinetic effects, including cyclotron dissipation and kinetic instabilities (micro-scale physics) adjust the temperature anisotropy in the slow winds.

In the present paper we investigate the instability of the electromagnetic ion-cyclotron (EMIC) modes, which may be driven by the temperature anisotropy of protons, $T_{\mathrm{p}, \perp}>T_{\mathrm{p}, \|}$ (Gary 1993; Gary et al. 1997, and the references therein) and exhibits a maximum growth rate at propagation parallel to the uniform magnetic field, i.e., $\boldsymbol{k} \times \boldsymbol{B}_{\mathbf{0}}=0$ (Kennel \& Petschek 1966). The constraining role of this instability on the solar wind proton anisotropy is still controversial since the $1 \mathrm{AU}$ measurements do not conform to the instability thresholds predicted by the existing theories (Hellinger et al. 2006; Bale et al. 2009). Hellinger et al. (2006) suggested that this disagreement may be explained by the simplifying assumptions on the plasma composition and particles distribution functions, or the nonlinear effects. For instance, the existence of alpha particles may considerably improve the agreement between the instability thresholds and the solar wind data (Maruca et al. 2012; Matteini et al. 2012). Moreover, resonant cyclotron interaction of EMIC modes with protons depends strongly on the specific shape of their velocity distribution (Isenberg et al. 2013), and in the solar wind this shape changes significantly in the presence of suprathermal populations and field-aligned beams. It then becomes clear that only a refined plasma description may provide a realistic linear prediction of observed data, since all the parameters, as the plasma composition, temperature ratios, anisotropy, and drift velocities of each species can introduce major effects in the dispersion and linear stability.

Cyclotron instabilities driven by the temperature anisotropy were first examined by Harris (1961) and Sagdeev \& Shafranov (1961), and today their study extends to a wide variety of plasma regimes conditioned by the kinetic anisotropy of plasma particles (Schlickeiser \& Skoda 2010; Maruca et al. 2012; Zhou et al. 2013; Chen et al. 2014; Gary et al. 2014; Omidi et al. 2014; Viňas et al. 2015). Only a detailed characterization of these instabilities allows a realistic estimation of their implication in different applications from space plasmas. Thus, cyclotron instabilities, especially the EMIC instability discussed here, are appreciated as a plausible candidate for explaining the enhanced fluctuations observed in the solar wind (Jian et al. 2009) and terrestrial magnetosphere (Nguyen et al. 2007). The interaction of the EMIC modes with plasma particles, including instability effects, but also particle scattering and wave-energy dissipation, is an interesting topic, with extended applications in astrophysical plasmas, mainly based on the ability of these left-handed (LH) polarized waves to resonate with protons and ions. However, more intriguing is the 
fact that the electrons may also be trapped or accelerated by the EMIC waves (Temerin \& Lysak 1984).

In the opposite situation when plasma particles exhibit an excess of parallel temperature $\left(T_{\perp}<T_{\|}\right)$, the firehose instability may develop with two distinct branches, namely, parallel and oblique. Of these, the oblique firehose is aperiodic, presumably faster, and with thresholds that better shape the limits of the solar wind temperature anisotropy (Hellinger et al. 2006; Štverák et al. 2008). However, this instability can accumulate the effects of both plasma species, electrons, and protons (Kennel \& Scarf 1968; Pilipp \& Völk 1971), and recently, Michno et al. (2014) have shown that anisotropic electrons may have an important influence on the parallel proton firehose (PFH) instability. Thus, for electrons with $T_{\mathrm{e}, \perp}>T_{\mathrm{e}, \|}$ the instability thresholds are found to approach the observed limits of the temperature anisotropy in the solar wind, reviving the potential role of the parallel firehose in constraining the solar wind anisotropy.

To our knowledge, the impact of electrons on the EMIC instability was almost completely ignored, motivated by the notion that the electrons are nonresonant, and the growth rates are essentially independent of the electron-proton temperature ratio $T_{\mathrm{e}} / T_{\mathrm{p}}$ (Gary 1993). However, the nonresonant destabilizing effects of electrons should also be taken into account, especially when an excess of parallel temperature $T_{\mathrm{e}, \perp}<T_{\mathrm{e}, \|}$ may drive unstable the so-called electron firehose (EFH) modes with frequencies in the range of ion gyrofrequency, and with the same LH polarization as the EMIC modes (Pilipp \& Völk 1971). We therefore aim to continue this series of investigations on the interplay of electrons and protons, and in this paper we focus the analysis on the EMIC instability and its potential implication in constraining the solar wind proton temperature anisotropy.

Our analysis starts in Section 2, beginning with the general dispersion relation for the parallel electromagnetic (LH polarized) modes in magnetized bi-Maxwellian-distributed plasmas, which assumes that both species of plasma particles are anisotropic (heavier ions are ignored, enabling us to isolate the effects of anisotropic electrons). Section 3 contains a detailed analysis of the unstable solutions, examining the effect of electrons on the growth rate, real frequency, and threshold conditions of the EMIC instability for two distinct situations given by the electron anisotropy, namely, $A_{\mathrm{e}}=T_{\mathrm{e}, \perp} / T_{\mathrm{e}, \|}>1$ or $A_{\mathrm{e}}<1$. Both of these two cases are investigated because a statistical analysis that correlates the anisotropy of protons (in our case, $A_{\mathrm{p}}=T_{\mathrm{p}, \perp} / T_{\mathrm{p}, \|}>1$ ) and the anisotropy of electrons from solar wind observations does not exist. The results of the present study are summarized in Section 4.

\section{PARALLEL TRANSVERSE MODES}

We consider a collisionless plasma of electrons $(\alpha=\mathrm{e})$ and protons $(\alpha=\mathrm{p})$, bi-Maxwellian distributed

$$
F_{\alpha}^{0}\left(v_{\|}, v_{\perp}\right)=\frac{1}{(2 \pi)^{3 / 2} u_{\alpha, \perp}^{2} u_{\alpha, \|}} \exp \left(-\frac{v_{\|}^{2}}{2 u_{\alpha, \|}^{2}}-\frac{v_{\perp}^{2}}{2 u_{\alpha, \perp}^{2}}\right),
$$

where $\omega$ is the wave-frequency, and $u_{\alpha, \|}=\sqrt{k_{B} T_{\alpha, \|} / m_{\alpha}}$ and $u_{\alpha, \perp}=\sqrt{k_{B} T_{\alpha, \perp} / m_{\alpha}}$ are thermal speeds, respectively, parallel and perpendicular to the stationary magnetic field $\boldsymbol{B}_{\mathbf{0}}$, defined by the corresponding temperatures $T_{\|}$, and $T_{\perp}$.
The dispersion relations of the transverse modes propagating parallel to the stationary magnetic field read (Gary 1993)

$$
\begin{aligned}
D^{ \pm}(k, \omega)= & 1-\frac{c^{2} k^{2}}{\omega^{2}}+\sum_{\alpha} \frac{\omega_{\mathrm{p}, \alpha}^{2}}{\omega^{2}}\left[\frac{\omega}{k u_{\|, \alpha}} Z^{0}\left(\xi_{\alpha}^{ \pm}\right)\right. \\
& \left.+\left(A_{\alpha}-1\right)\left\{1+\xi_{\alpha}^{ \pm} Z^{0}\left(\xi_{\alpha}^{ \pm}\right)\right\}\right]=0
\end{aligned}
$$

where $k$ is the wave-number, $c$ is the light speed, $\omega_{\mathrm{p}, \alpha}^{2}=4 \pi n_{\alpha} e^{2} / m_{\alpha}$ is the plasma frequency, \pm denote, respectively, the circular right-handed (RH) and $\mathrm{LH}$ polarization, $\Omega_{\alpha}=q_{\alpha} \boldsymbol{B}_{\mathbf{0}} / m_{\alpha} c$ is the gyrofrequency (nonrelativistic), and $A_{\alpha}=T_{\alpha, \perp} / T_{\alpha, \|}$ is the temperature anisotropy, and

$$
Z^{0}\left(\xi_{\alpha}^{ \pm}\right)=\frac{1}{\pi^{1 / 2}} \int_{-\infty}^{\infty} \frac{\exp \left(-x^{2}\right)}{x-\xi_{\alpha}^{ \pm}} d t, \Im\left(\xi_{\alpha}^{ \pm}\right)>0
$$

is the plasma dispersion function (Fried \& Conte 1961) of the argument

$$
\xi_{\alpha}^{ \pm}=\frac{\omega \pm \Omega_{\alpha}}{k u_{\alpha, \|}} .
$$

For the LH EMIC mode with $\omega<\Omega_{\mathrm{p}}$, the dispersion relation Equation (2) simplifies, in terms of the normalized quantities, to

$$
\begin{aligned}
& A_{\mathrm{p}}-1-\tilde{k}^{2}+\left(A_{\mathrm{p}} \frac{(\tilde{\omega}-1)}{\tilde{k} \sqrt{\beta_{\mathrm{p}, \|}}}+\frac{1}{\tilde{k} \sqrt{\beta_{\mathrm{p}, \|}}}\right) \\
& \quad \times Z^{0}\left(\frac{\tilde{\omega}-1}{\tilde{k} \sqrt{\beta_{\mathrm{p}, \|}}}\right)+\mu\left(A_{\mathrm{e}}-1+\left(A_{\mathrm{e}} \frac{(\tilde{\omega}+\mu)}{\tilde{k} \sqrt{\mu \theta \beta_{\mathrm{p}, \|}}}\right.\right. \\
& \left.\left.\quad-\frac{\mu}{\tilde{k} \sqrt{\mu \theta \beta_{\mathrm{p}, \|}}}\right) Z^{0}\left(\frac{\tilde{\omega}+\mu}{\tilde{k} \sqrt{\mu \theta \beta_{\mathrm{p}, \|}}}\right)\right)=0,
\end{aligned}
$$

where $\tilde{\omega}=\omega / \Omega_{\mathrm{p}}$ is the normalized frequency, $\tilde{k}=k c / \omega_{\mathrm{p}, \mathrm{p}}$ is the normalized wave-number, $\beta_{\mathrm{p}, \|}=8 \pi n_{\mathrm{e}} k_{B} T_{\mathrm{p}, \|} / B_{0}^{2}$ is the parallel proton plasma beta, $\mu=m_{\mathrm{p}} / m_{\mathrm{e}}$ is the proton/electron mass ratio, and $\theta=T_{\mathrm{e}, \|} / T_{\mathrm{p}, \|}$ is the electron/proton parallel temperature ratio.

\section{THE UNSTABLE EMIC SOLUTIONS}

In this section we analyze the unstable EMIC solutions and quantify the influence of the electrons anisotropy on the growth rate, real frequency, and threshold conditions for two distinct situations, conditioned by the electron anisotropy, i.e., $A_{\mathrm{e}}>1$, and $A_{\mathrm{e}}<1$. In order to perform this analysis the general dispersion relation (4) is solved exactly numerically.

\subsection{Electrons with $A_{\mathrm{e}}>1$}

The electron influence on the EMIC instability is first examined for the case when the electron anisotropy is manifested as an excess of perpendicular temperature $A_{\mathrm{e}}=T_{\perp, \mathrm{e}} / T_{\|, \mathrm{e}}>1$. The growth rate and the wave-frequency of the unstable solutions are displayed in Figures 1 and 2, respectively, for the same proton anisotropy $A_{\mathrm{p}}=T_{\perp, \mathrm{p}} / T_{\|, \mathrm{p}}=2$ and proton parallel beta $\beta_{\mathrm{p}, \|}=1$. Solutions are derived for different values of electron anisotropy $A_{\mathrm{e}}=1,1.5,2$, and temperature ratios $\theta=1,3,5$. Both the 

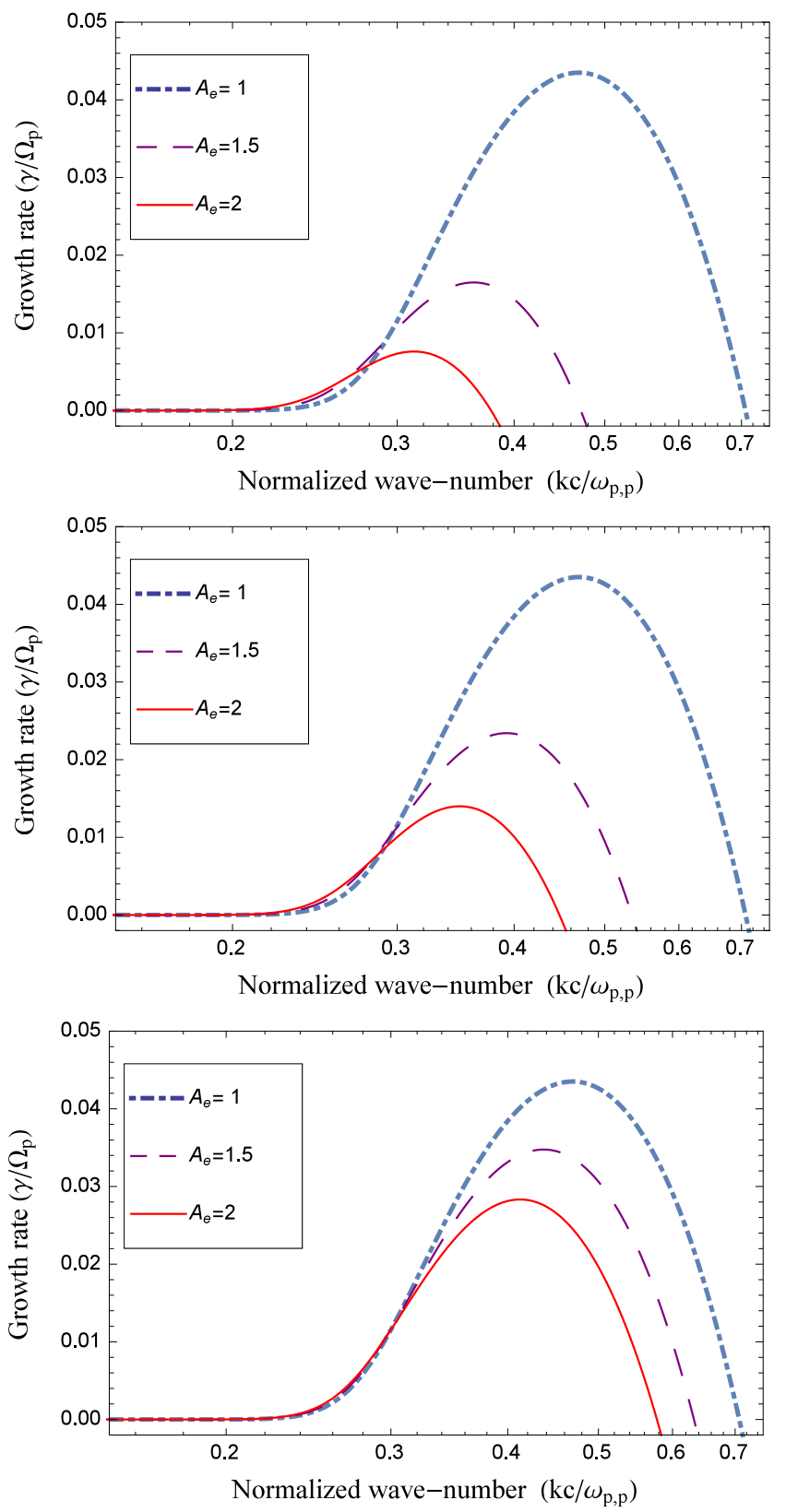

Figure 1. Effect of the electron anisotropy $A_{\mathrm{e}}=1,1.5,2$ and temperature ratio $\theta=5$ (top), 3 (middle), 1 (bottom) on the growth rates of EMIC instability with $A_{\mathrm{p}}=2, \beta_{\mathrm{p}, \|}=1$.

temperature anisotropy and the parallel plasma beta $\left(\beta_{\mathrm{e}, \|}=\theta \beta_{\mathrm{p}, \|}\right)$ for electrons are taken in the limits indicated by the solar wind observations (Štverák et al. 2008). Values chosen for the temperature ratio $\theta$ are also typical for the solar wind (Newbury et al. 1998; Stix 2012). If $A_{\mathrm{e}}>1$ is high enough in this case the electrons may drive an instability of the whistler (or electron-cyclotron) modes (Gary \& Karimabadi 1996). Protons do not react to these high-frequency fluctuations that grow much faster than the EMIC, but electrons are scattered and their temperature anisotropies are reduced below the instability thresholds, just under the limits indicated by the solar wind observations (Gary \& Wang 1996; Štverák et al. 2008). The peak of the growth rate decreases with the electron anisotropy (Figure 1), and this effect is enhanced by the temperature ratio $\theta$ (top panel). In this case we find that the
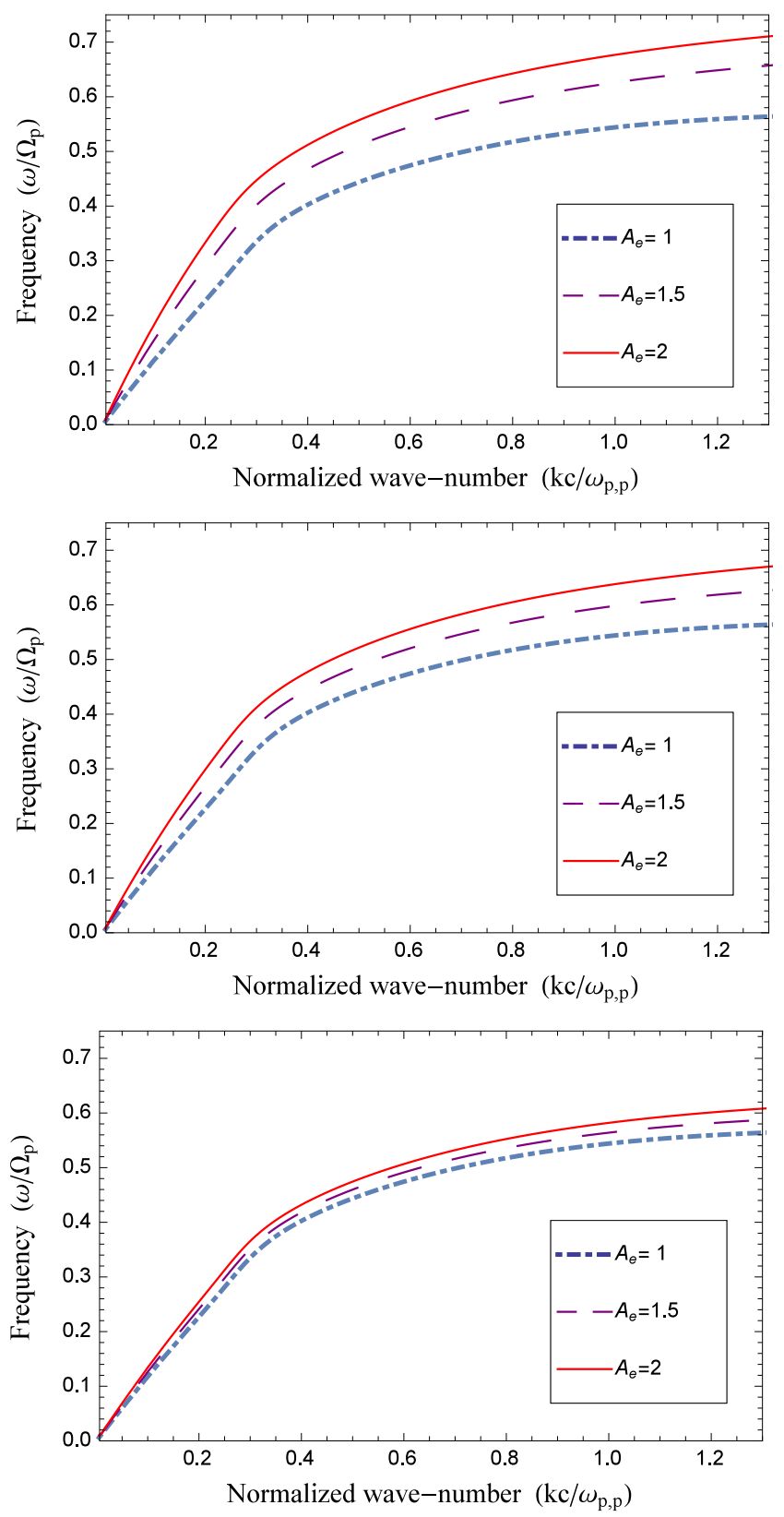

Figure 2. Effect of the electron anisotropy $A_{\mathrm{e}}=1,1.5,2$ and temperature ratio $\theta=5$ (top), 3 (middle), 1 (bottom) on the real frequencies of EMIC wave instability with $A_{\mathrm{p}}=2, \beta_{\mathrm{p}, \|}=1$.

electrons have an inhibiting effect on the EMIC instability. Otherwise, the real frequency of the EMIC modes increases with the electron anisotropy (Figure 2) and with the temperature ratio $\theta$ (top panel).

To present a more general picture of the electron effects, we analyze the anisotropy thresholds of the EMIC instability for two lower levels of the maximum growth rates $\gamma_{m}=10^{-3} \Omega_{\mathrm{p}}$ (Figure 3) and $\gamma_{m}=10^{-2} \Omega_{\mathrm{p}}$ (Figure 4). These are isocontours of the proton anisotropy versus proton parallel beta, calculated for different values of the electron anisotropy $A_{\mathrm{e}}=1,1.5,2$, and temperature ratio $\theta=1,3,5$. The anisotropy thresholds are calculated for an extended range of the plasma beta parameter $0.01<\beta_{\mathrm{p}, \|}<100$, including the solar wind and magnetospheric plasma conditions. Contours of the maximum growth rates are fitted to an inverse correlation law between 

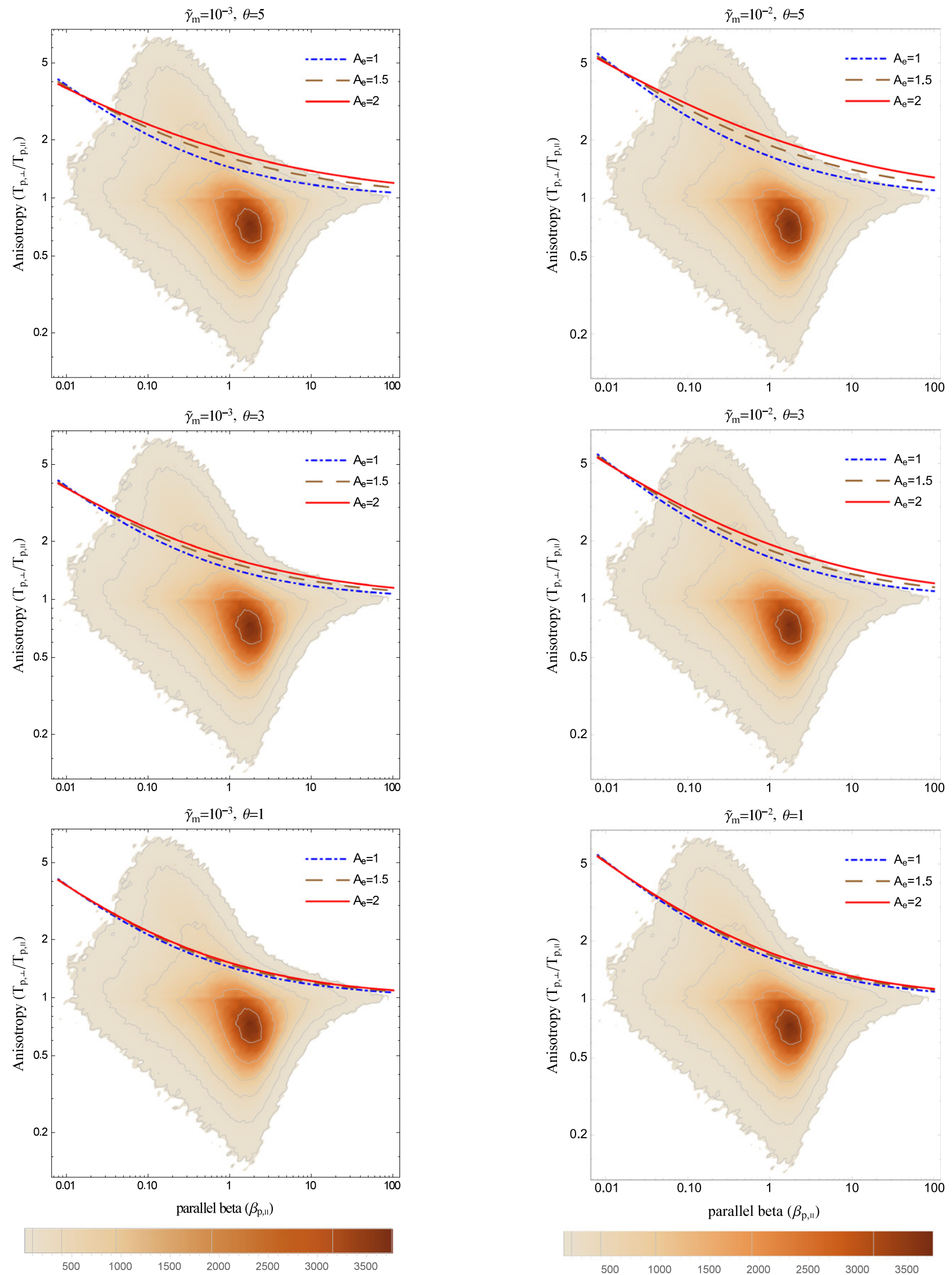

Figure 3. Effect of the electron anisotropy $A_{\mathrm{e}}=1,1.5,2$ and temperature ratio $\theta=5$ (top), 3 (middle), 1 (bottom) on the the threshold conditions of EMIC instability with the maximum growth rate $\gamma_{m} / \Omega_{\mathrm{p}}=10^{-3}$.

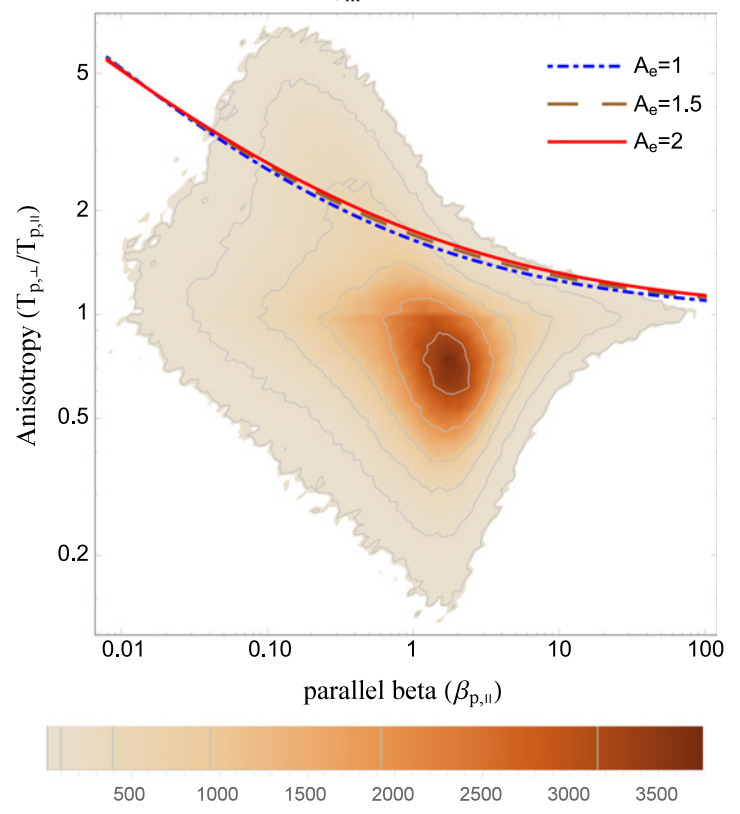

Figure 4. Effect of the electron anisotropy $A_{\mathrm{e}}=1,1.5,2$ and temperature ratio $\theta=5$ (top), 3 (middle), 1 (bottom) on the threshold conditions of EMIC instability with the maximum growth rate $\gamma_{m} / \Omega_{\mathrm{p}}=10^{-2}$. 
Table 1

Proton Anisotropy Fitting Parameters

\begin{tabular}{ccccccc}
\hline \hline & & \multicolumn{2}{c}{$\gamma_{m} / \Omega_{\mathrm{p}}=10^{-3}$} & & \multicolumn{2}{c}{$\gamma_{m} / \Omega_{\mathrm{p}}=10^{-2}$} \\
\cline { 3 - 4 }$\theta$ & $A_{\mathrm{e}}$ & $a$ & $b$ & & $a$ & $b$ \\
\hline 5 & 2 & 0.7378 & 0.2832 & 1.0568 & 0.2884 \\
& 1.5 & 0.6194 & 0.3272 & 0.8737 & 0.3341 \\
3 & 2 & 0.6426 & 0.3177 & & 0.9164 & 0.3227 \\
& 1.5 & 0.5554 & 0.3518 & & 0.7899 & 0.3582 \\
1 & 2 & 0.5242 & 0.3665 & & 0.7469 & 0.3700 \\
& 1.5 & 0.4890 & 0.3824 & & 0.7008 & 0.3854 \\
& 1 & 0.4465 & 0.4032 & & 0.6433 & 0.4058 \\
& & & & &
\end{tabular}

temperature anisotropy, $A_{\mathrm{p}}$, and plasma beta, $\beta_{\mathrm{p}, \|}$ (Gary \& Lee 1994)

$$
A_{\mathrm{p}}=1+\frac{a}{\beta_{\mathrm{p}, \|}^{b}},
$$

where the fitting parameters $a$ and $b$ are tabulated in Table 1. If $\beta_{\mathrm{p}, \|}>0.05$ is large enough, the anisotropy thresholds are increased by the electron anisotropy, confirming the same effect described above. Otherwise, the effect may be the opposite for very low values of $\beta_{\mathrm{p}, \|}$, but in these regimes the parallel cyclotron modes may compete with the electrostatic oblique instabilities (Gary et al. 2011). These effects are again found to be enhanced by the temperature ratio $\theta$, but it is worth noting that the influence of the temperature ratio $\theta$ vanishes for $A_{\mathrm{e}}=1$, in accordance with previous studies which were limited to the study of isotropic electrons (Gary 1993).

These thresholds are contrasted with the 10-year observational data of the proton anisotropy measured at $1 \mathrm{AU}$ in the solar wind as a function of the proton parallel beta. Provided by WIND/SWE and MFI instruments for the proton velocity distribution and magnetic field, respectively (Lepping et al. 1995; Ogilvie et al. 1995; Bale et al. 2009), these data are displayed in Figures 3 and 4 with a color logarithmic scale representing the number of events (only for bins with more than 20 events). In the absence of a Coulomb relaxation the excess of perpendicular temperature, i.e., $A_{\mathrm{p}}>1$ in the upper part of these diagrams, is expected to be constrained by the EMIC instability, such that the limits of the observations are well shaped by the instability thresholds. Previous calculations have only assumed that isotropic electrons and the resulting EMIC thresholds do not show a good fit to the observations, but a slope lower than the observed limits of the temperature anisotropy (Hellinger et al. 2006). Here the EMIC thresholds are derived considering the electrons that are anisotropic with $A_{\mathrm{e}}>1$, and their slopes are found to be even lower, departing from the observational limits of the proton anisotropy. For higher values of the (parallel) beta parameter, the instability is inhibited by the electron anisotropy and the instability thresholds increase.

\subsection{Electrons with $A_{\mathrm{e}}<1$}

As mentioned in the introduction, it is probably more reasonable to study the opposite case when the electrons exhibit an excess of parallel temperature, i.e., $A_{\mathrm{e}}<1$, and when the electrons are expected to stimulate the destabilizing effect on the EMIC modes. Figures 5 and 6 display the growth rate and the real frequency of the EMIC instability for
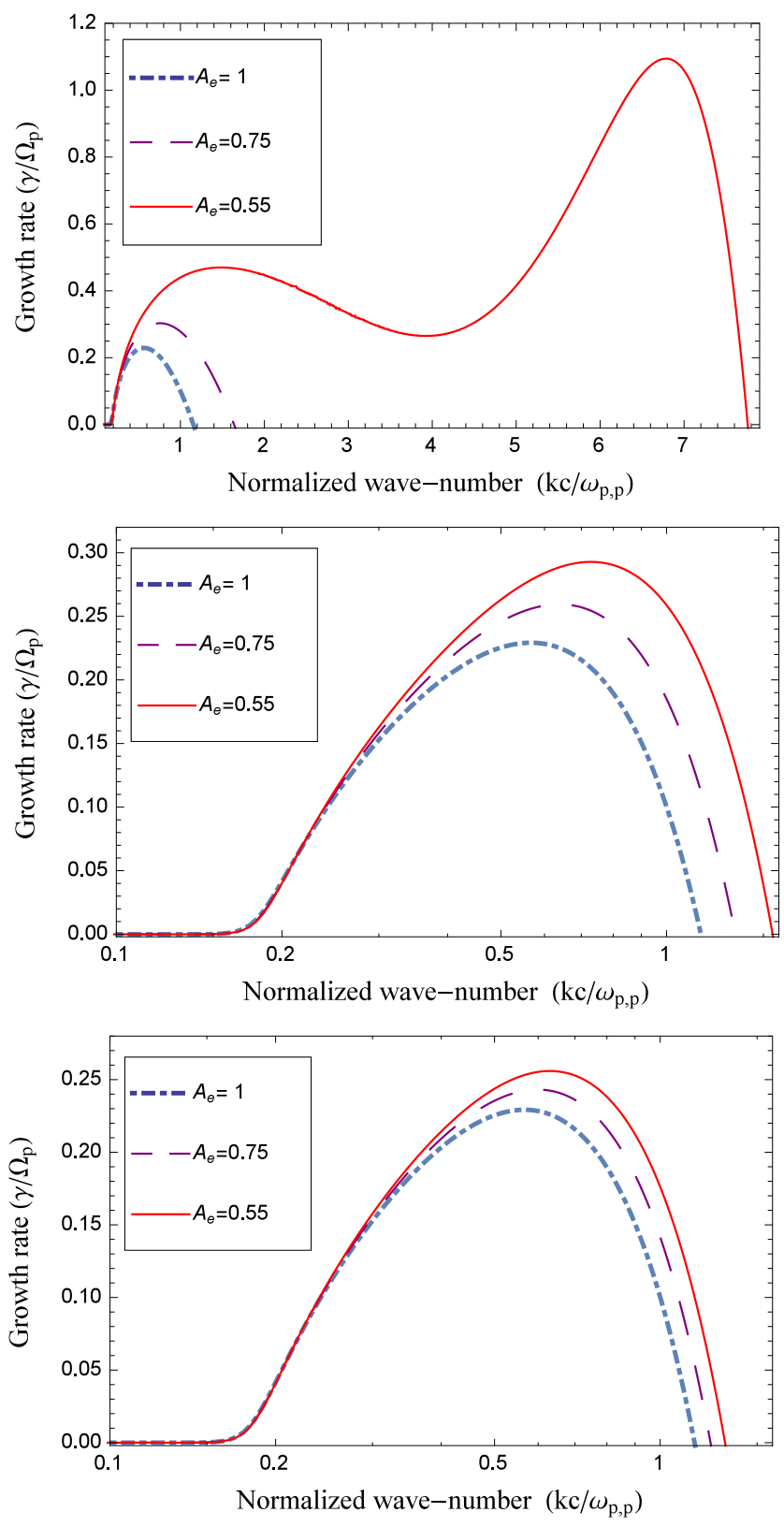

Figure 5. Effect of the electron anisotropy $A_{\mathrm{e}}=1,0.75,0.55$ and temperature ratio $\theta=2$ (top), 1 (middle), 0.5 (bottom) on the growth rates of EMIC instability for $A_{\mathrm{p}}=3, \beta_{\mathrm{p}, \|}=2$.

$A_{\mathrm{p}}=3, \beta_{\mathrm{p}, \|}=2$, and compare them for different electron anisotropies $A_{\mathrm{e}}=1,0.75,0.55$, and different temperature ratios $\theta=0.5,1,2$. Confirming the expectation, the EMIC peaks are indeed increased by the electron anisotropy $A_{\mathrm{e}}<1$, and the temperature ratio $\theta$ enhances this effect.

Furthermore, with suitable values of $A_{\mathrm{e}}$ and $\theta$, growth rates of the unstable solution may display two distinct peaks; see the top panel in Figure 5 for $A_{\mathrm{e}}=0.55, \theta=2$. The first peak at low wave-numbers corresponds to the LH EMIC mode, and the second peak raises at larger wave-numbers due to the EFH instability. The corresponding wave-frequency plotted in Figure 6 remains positive, clearly indicating that these two modes have the same LH polarization. These results are similar to those obtained by Michno et al. (2014) for the interplay of the PFH instability with EFH instability. They also found two 

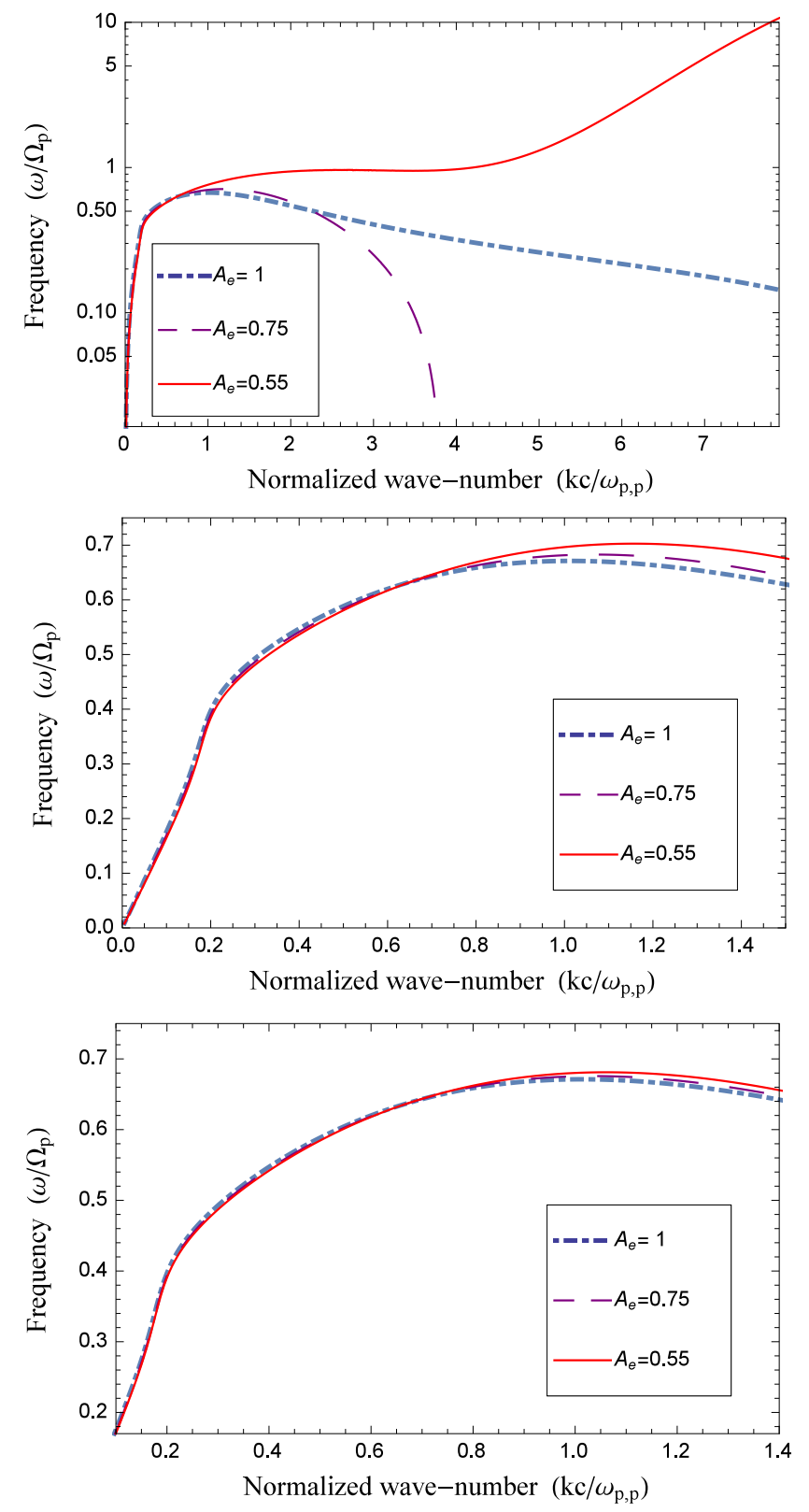

Figure 6. Effect of the electron anisotropy $A_{\mathrm{e}}=1,0.75,0.55$ and temperature ratio $\theta=2$ (top), 1 (middle), 0.5 (bottom) on the real frequencies of EMIC instability for $A_{\mathrm{p}}=3, \beta_{\mathrm{p}, \|}=2$.

peaks but the wave-frequency changes the sign according to the opposite polarizations of the EFH and PFH modes. In our case, the interplay of the EMIC and EFH can produce two peaks only for a sufficiently large anisotropy of the electrons exceeding thresholds of the EFH instability. This result seems to be in agreement with the 1D PIC simulations, which suggest that the $\mathrm{LH}$ polarized $\mathrm{EFH}$ modes may trigger an efficient resonant transfer of energy to a large fraction of protons, enhancing their anisotropy $T_{\mathrm{p}, \perp}>T_{\mathrm{p}, \|}$ (Messmer 2002) and explaining a subsequent stimulation of the EMIC instability, if the EFH is fast enough (e.g., Figure 5, with a peak higher than the EMIC). However, we are aware of the fact that the electron effects may be overestimated in this case by considering only the parallel (or the nonresonant, cf., Gary \& Nishimura 2003) branch of the EFH instability. For a wide range of parameters
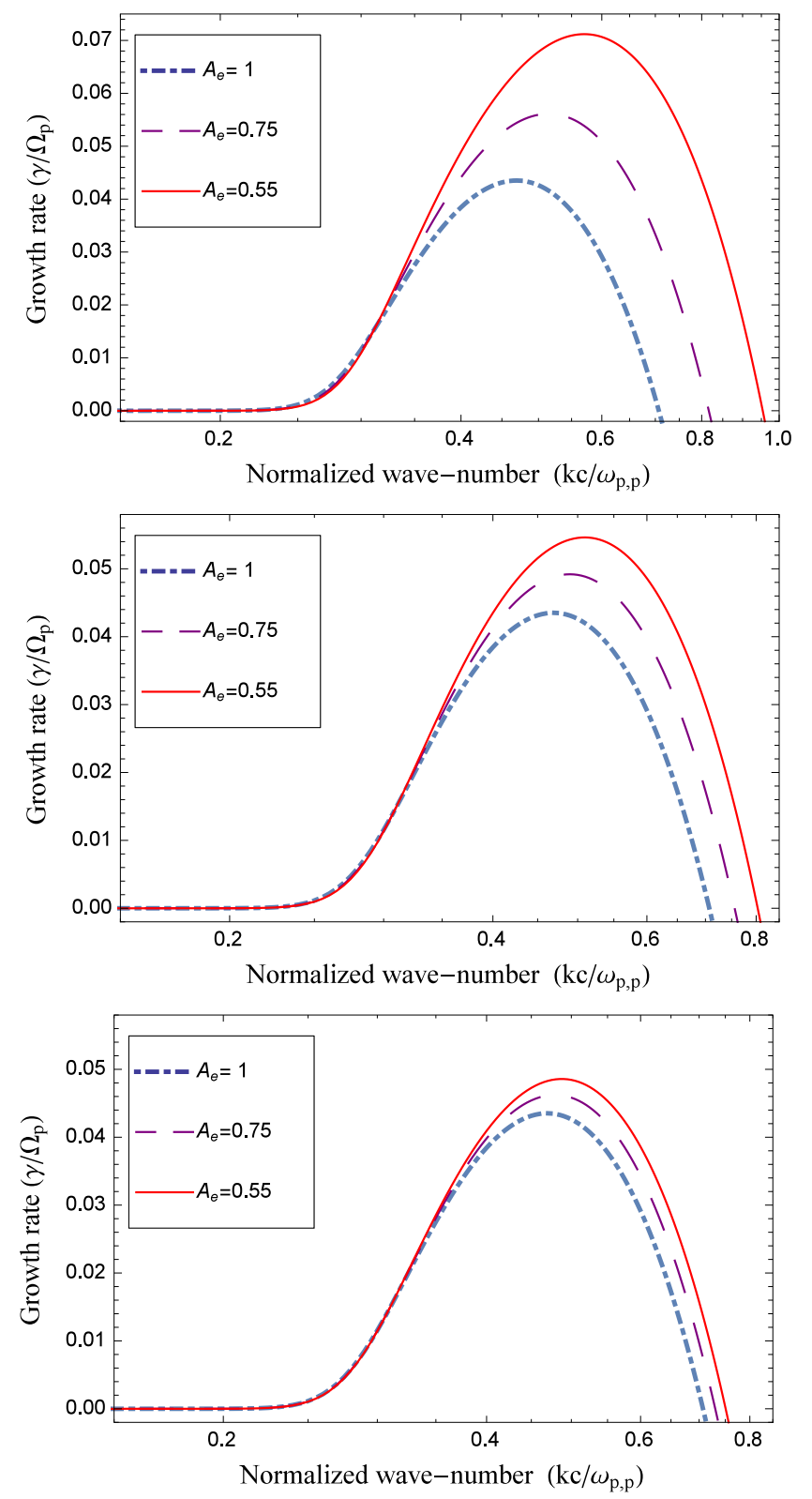

Figure 7. Effect of the electron anisotropy $A_{\mathrm{e}}=1,0.75, .55$ and temperature ratio $\theta=2$ (top), 1 (middle), 0.5 (bottom) on the growth growth rates of EMIC instability for $A_{\mathrm{p}}=2, \beta_{\mathrm{p}, \|}=1$.

relevant to the solar wind, the oblique (or resonant) EFH has lower thresholds and reaches maximum growth rates (comparable to the electron gyrofrequency) that are much higher than the parallel EFH (with maximum growth rates comparable to the proton gyrofrequency). The EFH peak in Figure 5 may therefore not be realistic due to oblique EFH instability that develops faster, spending the electron free energy, but probably without much influence on protons (Gary \& Nishimura 2003; Camporeale \& Burgess 2008) and the EMIC instability.

In addition, the analysis may be extended, like, for instance, the cases in Figures 7 and 8, where we consider the same $A_{\mathrm{e}}=1,0.75,0.55$ but lower values for the proton parameters $A_{\mathrm{p}}=2, \beta_{\mathrm{p}, \|}=1$, such that, for a temperature ratio $\theta=2,1$, 0.5 , the electron plasma beta $\beta_{\mathrm{e}, \|} \leqslant 2$ becomes too small to ensure conditions for any of the oblique or parallel firehose to 

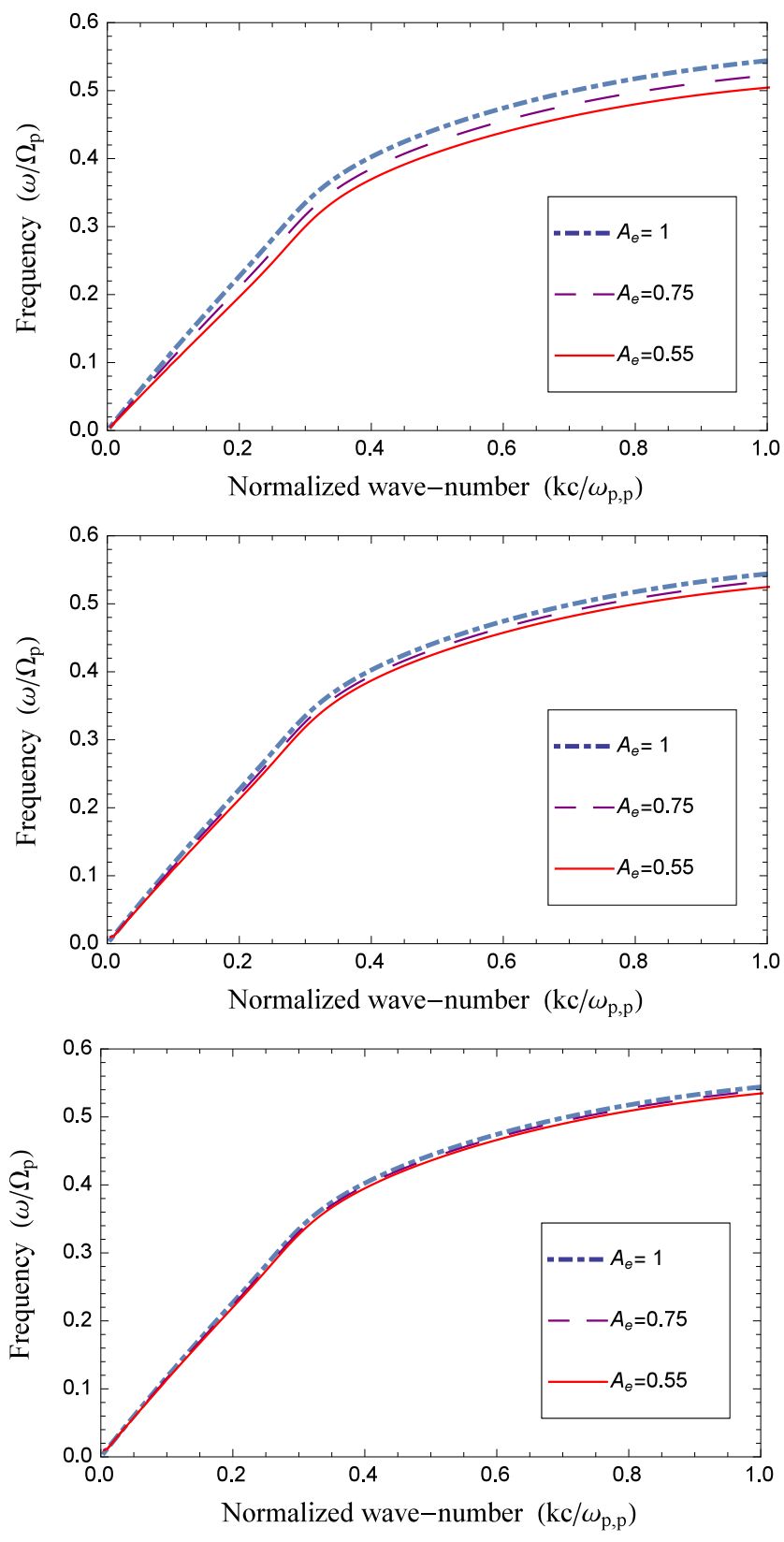

Figure 8. Effect of the electron anisotropy $A_{\mathrm{e}}=1,0.75, .55$ and temperature ratio $\theta=2$ (top), 1 (middle), 0.5 (bottom) on the real frequencies of EMIC instability for $A_{\mathrm{p}}=2, \beta_{\mathrm{p}, \|}=1$.

develop. However, the anisotropic electrons induce the same effect on the EMIC growth rates and wavefrequencies.

In the same order, Figure 9 presents the anisotropy thresholds as isocontours of the maximum growth rates at a lower level $\gamma_{m} / \Omega_{\mathrm{p}}=10^{-3}$. We use the same technique described above to produce these isocontours by fitting the instability thresholds derived numerically with the same law in Equation (5). The fitting parameters $a$ and $b$ are tabulated in Table 2. To study the electron effect on the EMIC instability we consider the same sets of values for the electron anisotropy $A_{\mathrm{e}}=1,0.75,0.55$ and temperature ratio $\theta=0.5,1,2$. The same enhancing effect on the instability growth rates is shown by the instability thresholds, which take lower values as the electron anisotropy increases, and this effect is enhanced by increasing $\theta$.
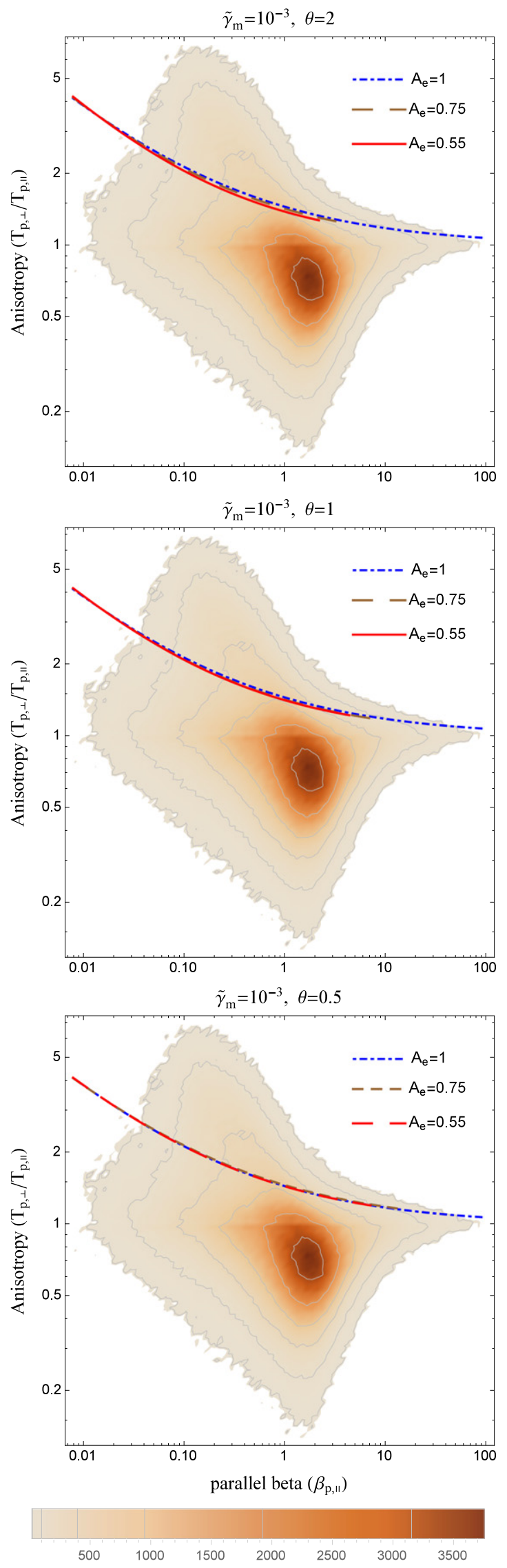

Figure 9. Effect of the electron anisotropy $A_{\mathrm{e}}=1,0.75,0.55$ and temperature ratio $\theta=2$ (top), 1 (middle), 0.5 (bottom) on the threshold conditions of EMIC instability with maximum growth rate $\gamma_{m} / \Omega_{\mathrm{p}}=10^{-3}$. 
Table 2

Proton Anisotropy Fitting Parameters

\begin{tabular}{lccc}
\hline \hline & & \multicolumn{2}{c}{$\gamma_{m} / \Omega_{\mathrm{p}}=10^{-3}$} \\
\cline { 3 - 4 }$\theta$ & $A_{\mathrm{e}}$ & $a$ & $b$ \\
\hline 2 & 0.55 & 0.382 & 0.439 \\
& 0.75 & 0.421 & 0.416 \\
1 & 0.55 & 0.412 & 0.422 \\
& 0.75 & 0.420 & 0.417 \\
0.5 & 0.55 & 0.447 & 0.402 \\
& 0.75 & 0.465 & 0.393 \\
& 1 & 0.448 & 0.402 \\
\hline
\end{tabular}

Differences introduced by the electron anisotropy are hardly visible in Figure 9, indicating a negligible influence on the instability thresholds at these low levels. However, the derivation of the EMIC instability thresholds is conditioned in this case by the parametric range where we can distinguish between the peaks of the EMIC and EFH growth rates, this range being limited by the large values of $\beta_{\mathrm{e}, \|}=\theta \beta_{\mathrm{p}, \|}>1$, when the EFH peak dominates and the EMIC peak cannot be distinguished. The maximum cutoff values of the proton parameters $\beta_{\mathrm{p}, \|}$ and $A_{\mathrm{p}}$, where the EMIC still exhibits a distinct peak are listed in Table 3 . The effects of the anisotropic electrons on the instability thresholds may be enhanced by increasing the temperature ratio $\theta$, but this will reduce the cutoff for $\beta_{\mathrm{p}, \|}$ and implicitly the relevance of the instability thresholds. It then becomes clear that the instability thresholds do not improve their alignment to the observations.

\section{SUMMARY}

In the present paper we have refined the analysis of the EMIC instability, assuming that both species, the electrons and protons, are anisotropic, and considering their cumulative effect to produce a realistic model. According to our knowledge, such an analysis of the EMIC instability was not reported in the literature before, and if then only for isotropic electrons. The growth rate, real frequency, and threshold conditions are here found to be highly sensitive to the electron temperature anisotropy with respect to the background magnetic field.

The electrons with an excess of perpendicular temperature, i.e., $A_{\mathrm{e}}>1$, have an inhibiting effect on the EMIC growth rates, which decrease with increasing electron anisotropy, while the wave-frequency is increased. Kennel \& Scarf (1968) offered an original explanation that these anisotropic electrons may change the wave-frequency of the EMIC modes and implicitly the number of resonant protons that trigger the EMIC instability. In our case the wave-frequency and implicitly the wave-phase speed are increased, causing the number of resonant protons to decrease, and thus explaining the inhibiting effect on the instability. This effect is also confirmed by the anisotropy thresholds, which increase with the electron anisotropy. Compared with the observations in the solar wind, the instability thresholds obtained in this case do not show a better alignment to the margins of the proton anisotropy measured at $1 \mathrm{AU}$; their slopes are found to be even lower than those obtained for isotropic electrons $A_{\mathrm{e}}=1$, especially for high values of the parallel beta $\beta_{\mathrm{p}, \|}$.

In the opposite case, the anisotropic electrons with $A_{\mathrm{e}}<1$ stimulate the destabilizing effect of the EMIC mode, enhancing the growth rates. However, the electron effects on the
Table 3

Cutoff Values of Proton Parameters $\beta_{\mathrm{p}, \|}, A_{\mathrm{p}}$

\begin{tabular}{lccc}
\hline \hline$A_{\mathrm{e}}$ & $\theta$ & $\beta_{\mathrm{p}, \|}$ & $A_{\mathrm{p}}$ \\
\hline 0.55 & 2 & 2.2 & 1.05 \\
& 1 & 4.4 & 1.042 \\
& 0.5 & 8.8 & 1.036 \\
0.75 & 2 & 3.9 & 1.055 \\
& 1 & 7.9 & 1.038 \\
& 0.5 & 13.9 & 1.0635 \\
\hline
\end{tabular}

instability thresholds are minor and do not improve their fit to observations. Highly anisotropic electrons can drive the EFH modes (parallel or oblique) to be unstable, giving rise, in the parallel direction, to a second peak of the growth rates at larger wave-numbers. If the parallel EFH instability is fast enough, it may be efficient in the exchange of energy between electrons and protons, explaining the enhancing effects of electrons on the EMIC instability. However, this hypothesis is undermined by the competition with the oblique EFH instability that usually is much faster than the parallel EFH, and may transfer energy to protons but mainly in a parallel direction (Gary \& Nishimura 2003; Camporeale \& Burgess 2008), inhibiting the EMIC modes.

The results of the present paper provide an improved, and tentatively more realistic model for studying the interplay of protons and electrons, and predicting their destabilizing effects on the EMIC modes. We have shown that the effect of electrons may be stimulated by their anisotropy as well as the electron-proton temperature ratio $\theta$. Realistic models are vital for understanding the role played by the wave-fluctuations in the collision-poor or collisionless plasmas from space. In these plasmas the Coulomb collisions are not effective to constrain departures from thermal equilibrium, but the instabilities and resulting fluctuations can ensure a self-regulation of the kinetic anisotropy in these plasmas. Based on our present results, we can conclude by strengthening previous findings (Hellinger et al. 2006; Bale et al. 2009) that the EMIC instability thresholds do not shape the limits of the proton anisotropy $A>1$ measured at $1 \mathrm{AU}$ in the solar wind. The role played by this instability in constraining the proton anisotropy therefore remains unclear (Isenberg et al. 2013), but it can be unveiled in a more extended and realistic manner by taking into account the additional effects of the competing instabilities, e.g., mirror instability, as well as the implication of suprathermal populations, which are ubiquitous in the solar wind. The effects of the suprathermal protons on the EMIC instability are known but only for isotropic electrons (Xiao et al. 2007; Lazar 2012; Lazar \& Poedts 2014). This analysis will be completed in the second paper of this series to include both the suprathermal and anisotropic electron effects.

The authors acknowledge the use of WIND SWE (Ogilvie et al. 1995) ion data, and WIND MFI (Lepping et al. 1995) magnetic field data from the SPDF CDAWeb service: http:// cdaweb.gsfc.nasa.gov/. The authors acknowledge support from the Katholieke Universiteit Leuven. These results were obtained in the framework of the projects GOA/2009-009 (KU Leuven), G.0729.11 (FWO-Vlaanderen), and C 90347 (ESA Prodex 9). The research leading to these results has also received funding from the European Commission's Seventh Framework Programme (FP7/2007-2013) under the grant 
agreements SOLSPANET (project n 269299, www.solspanet. eu), SPACECAST (project n 262468, fp7-spacecast.eu), eHeroes (project n 284461, www.eheroes.eu), and SWIFF (project n 263340, www.swiff.eu). S.M. Shaaban would like to thank the Egyptian Ministry of Higher Education for supporting his research activities and would like to acknowledge the discussions and suggestions of Prof. S. A. Elwakil.

\section{REFERENCES}

Bale, S. D., Kasper, J. C., Howes, G. G., et al. 2009, PhRvL, 103, 211101 Camporeale, E., \& Burgess, D. 2008, JGRA, 113, A07107

Chen, L., Jordanova, V. K., Spasojević, M., Thorne, R. M., \& Horne, R. B. 2014, JGRA, 119, 2963

Chew, G. F., Goldberger, M. L., \& Low, F. E. 1956, in Proc. RSL Conf. Ser. A, vol 236, Mathematical and Physical Science, 112

Fried, B. D., \& Conte, S. D. 1961, The Plasma Dispersion Function (New York: Academic)

Gary, S. P. 1993, Theory of Space Plasma Microinstabilities (Cambridge: Cambridge Univ. Press)

Gary, S. P., Hughes, R. S., Wang, J., \& Chang, O. 2014, JGRA, 119, 1429

Gary, S. P., \& Karimabadi, H. 1996, JGR, 111, A11224

Gary, S. P., \& Lee, M. A. 1994, JGRA, 99, 11297

Gary, S. P., Liu, K., \& Winske, D. 2011, PhPl, 18, 082902

Gary, S. P., \& Nishimura, K. 2003, PhPl, 10, 3571

Gary, S. P., \& Wang, J. 1996, JGRA, 101, 10749

Gary, S. P., Wang, J., Winske, D., \& Fuselier, S. A. 1997, JGRA, 102, 27159

Harris, E. G. 1961, J. Nucl. Energy, Part C, Plasma Phys., 2, 138
Hellinger, P., Trávníček, P., Kasper, J. C., \& Lazarus, A. J. 2006, GeoRL, 33, L09101

Isenberg, P. A., Maruca, B. A., \& Kasper, J. C. 2013, ApJ, 773, 164

Jian, L. K., Russell, C. T., Luhmann, J. G., et al. 2009, ApJL, 701, L105

Kennel, C. F., \& Petschek, H. E. 1966, JGRA, 71, 1

Kennel, C. F., \& Scarf, F. L. 1968, JGRA, 73, 6149

Lazar, M. 2012, A\&A, 547, A94

Lazar, M., \& Poedts, S. 2014, MNRAS, 437, 641

Lepping, R. P., Acǔna, M. H., Burlaga, L. F., et al. 1995, SSRv, 71, 207

Maruca, B. A., Kasper, J. C., \& Gary, S. P. 2012, ApJ, 748, 137

Matteini, L., Hellinger, P., Landi, S., Trávníček, P. M., \& Velli, M. 2012, SSRv, 172, 373

Matteini, L., Landi, S., Hellinger, P., et al. 2007, GeoRL, 34, L20105

Messmer, P. 2002, A\&A, 382, 301

Michno, M. J., Lazar, M., Yoon, P. H., \& Schlickeiser, R. 2014, ApJ, 781, 49

Newbury, J. A., Russell, C. T., Phillips, J. L., \& Gary, S. P. 1998, JGRA, 103, 9553

Nguyen, S. T., Perez, J. D., \& Fennell, J. F. 2007, JGR, 112, A12203

Omidi, N., Isenberg, P., Russell, C. T., Jian, L. K., \& Wei, H. Y. 2014, JGRA, 119,1442

Ogilvie, K. W., Chornay, D. J., Fritzenreiter, R. J., et al. 1995, SSRv, 71, 55 Pilipp, W., \& Völk, H. J. 1971, JPIPh, 6, 1

Sagdeev, R. Z., \& Shafranov, V. D. 1961, JETP, 12, 130

Schlickeiser, R., \& Skoda, T. 2010, ApJ, 716, 1596

Štverák, Š., Trávniíček, P., Maksimovic, M., et al. 2008, JGRA, 113, A03103

Stix, M. 2012, The Sun: An Introduction (Berlin: Springer)

Temerin, M., \& Lysak, R. L. 1984, JGRA, 89, 2849

Viňas, A. F., Moya, P. S., Navarro, R. E., et al. 2015, JGRA, 120, 3307

Xiao, F., Zhou, Q., He, H., Zheng, H., \& Wang, S. 2007, JGRA, 112, A07219

Zhou, Q., Xiao, F., Shi, J., et al. 2013, JGRA, 118, 340 